\title{
Particle Timing Control and Alignment in Microchannel Flow by Applying Periodic Force Control Using Dielectrophoretic Force
}

\author{
Kazuya Tatsumi*, Koki Kawano, Hiromichi Shintani and Kazuyoshi Nakabe \\ Department of Mechanical Engineering and Science, Kyoto University, \\ Kyotodaigakukatsura, Kyoto, Kyoto 615-8450, Japan.
}

Corresponding Author

*E-mail: tatsumi@me.kyoto-u.ac.jp

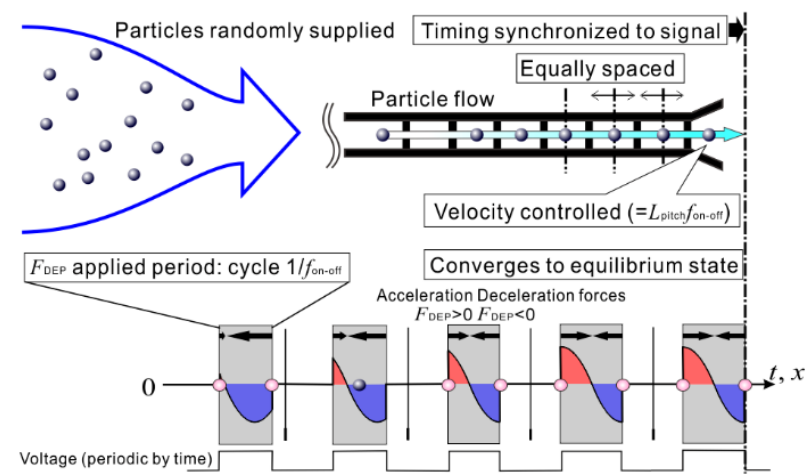

\section{Abstract}

In this study, a technique for particle streamwise timing, spacing and velocity control (alignment) in microchannel flow by controlling the forces exerted on the particle in space and time was developed. In the present technique, the timing of particles crossing a certain position in microchannel flow with specific interval, and the particle velocity are controlled by applying acceleration and deceleration forces periodically in the streamwise direction, and activating them periodically. The force is produced by dielectrophoretic force using ladder-type electrodes embedded in the microfluidic device, and is turned on and off in a cycle. The timing of particles crossing a certain position can be changed by adjusting the phase of the on-off cycle; i.e. the phase of the voltage signal. In the experiment, timing and velocity were measured at the inlet and outlet of ladder-type regions for Jurkat cells and particles of some variation in sizes, and probability density functions for the deviation of these values from the equilibrium (aligned) state were evaluated. Further, we will discuss the motion characteristics of the particles numerically and experimentally to understand the mechanism and evaluate the performance of the particle timing control and alignment using the present technique. The results confirm that the particles randomly distributed at the inlet of ladder-type electrode regions are controlled to flow with even spacing at a specific velocity. Moreover, the timing of the particles passing a specific location in the ladder-type electrode region was synchronized with the activated/non-activated cycle of the applied force and could be specified. 
Controlling the timing of the particles (or cells and droplets) passing a specific location in the microchannel flow is one of the important factors in microfluidics as timing and position control results in higher accuracy and performance in sensing, sorting, capsuling, fusion, and synthesis systems. In most cases, particles flow into the microchannel randomly as the particles and cells are randomly dispersed in the supplied fluid. Therefore, not only the position of the particles in all three Cartesian directions in the microchannel flow, but also the time when they cross a streamwise location are random and can affect the accuracy and throughput of the microfluidic system. One good example is active sorting systems, in which the sorting is performed by manipulating specific particles and cells from others by applying an external force. ${ }^{1-14}$ In this case, the performance relies not only on the position of the particles and cells but also the timing when the force is applied to them for manipulation. Further, for electric and optic sensors, the signal is based on the interference of particles and cells on the electric field and optical path, and controlling the timing and interval of the particles and cells contributes to increase the throughput and to reduce the performance requirements of the detector such as acquisition rate of the signals and images. ${ }^{15-18}$ For encapsulation of particles in droplets, the streamwise spacing of the particles and cells needs to be controlled so that the time interval of the particle and cell injection matches that of droplet generation. ${ }^{19-29}$ If the timing (phase) of the particles can be controlled in addition to the time interval, the accuracy can further increase especially when coupling/grouping more than several particles in a location or the droplet.

Numerous technologies have been developed to control and focus the particles position in the crosssectional plane of the channel. Convection of particles using secondary flows such as Dean flow and corner vortices can focus the particles to the stagnation region. ${ }^{30-32}$ Inertial microfluidics locates the particles where the lift force generated by shear flow and centrifugal forces is balanced. ${ }^{30,33-38}$ External forces such as electrophoretic and dielectrophoretic forces ${ }^{12,39-47}$, acoustic force $e^{48-50}$, and magnetic forces ${ }^{51}$ can be exerted on the particles to control their position. In non-Newtonian fluid flow, normal stresses generated additionally by the viscoelasticity of the non-Newtonian fluid flow produce elastic lift and drag forces that can focus the particles in the flow. ${ }^{52}$

Controlling the streamwise characteristics of particles in flow is more difficult as the relative position of each particle as it flows in the channel needs to be controlled. One such technique is inertial microfluidics, in which the streamwise spacing of the particles is controlled to form "trains" of particles. In this case, the flow generated upstream and downstream of each particle by its existence and secondary flows produce an additional force to accelerate or decelerate the particles to converge in equal spacing positions. ${ }^{34-37}$

While the time interval between the particles can be controlled by these techniques, however, the timing of the particles crossing/entering a specific location in the channel cannot be predicted or 


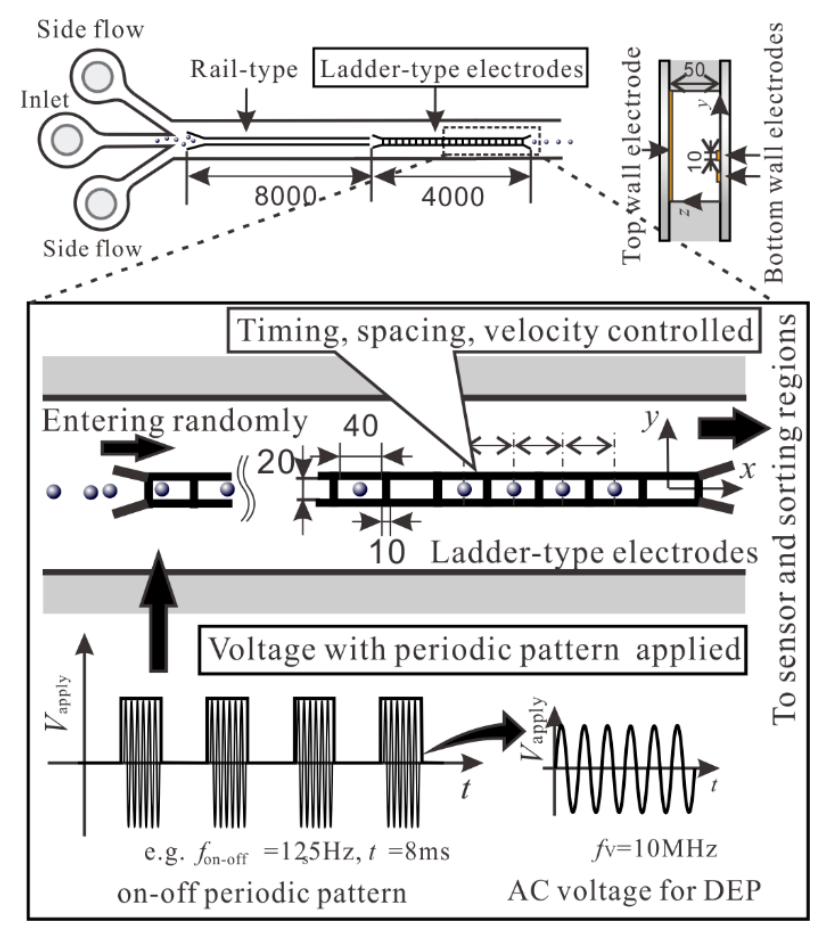

Figure 1: Schematic diagram of ladder-type (and rail-type) electrodes and microchannel in the microfluidic device. The rail-type electrode is applied to control spanwise and height position, and accurately guide particles to the inlet of the ladder-type electrodes.

controlled. As previously mentioned, knowing and controlling the timing of particles is one way to increase the performance or realize the sorting, sensing and other microfluidic systems. If we can specify the timing as well as the time interval and velocity or particles flowing in the microchannel, we can improve the performance of these devices.

We have developed a technique that can control the timing of the particles crossing a certain positon in the microchannel, and the spacing and velocity of particles simultaneously by applying the force exerted on particles with space and time. More specifically, dielectrophoretic (DEP) force is generated in periodic form in the streamwise direction by using ladder-shaped electrodes and this DEP force is changed periodically over time. A schematic diagram of the electrode patterns is shown in Fig. 1. The ladder-type electrodes periodically form areas in which particles accelerate and decelerate in the streamwise direction when alternating current voltage is applied to the electrodes. The supplied voltage is then turned on and off periodically with another frequency that differs from this high frequency voltage for AC type DEP force generation to apply force to particles periodically and intermittently with time. By these effects, particles flowing through the ladder-type electrodes region form a line with distance between the particles defined by the pitch of cross-tie electrodes as they flow downstream. Moreover, as the particle motion is synchronized with the periodic time distributions of voltage on-off, the time phase between the relative position of the particles and the periodic cycle of 


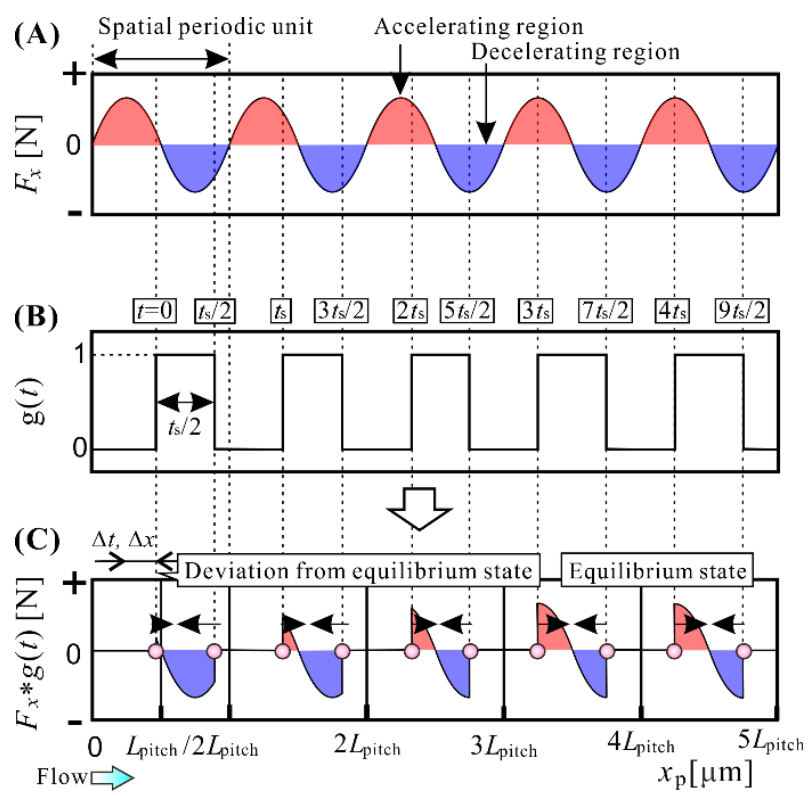

Figure 2. Dielectrophoretic (DEP) force $F_{\mathrm{DEP}}$ periodically varied in space and time is exerted on particles. (A) $F_{\text {DEP }}$ distributed periodically in the streamwise direction to accelerate and decelerate the particles. (B) On and off periodic time cycle of $F_{\text {DEP. }}(\mathrm{C})$ Actual force particle receives at a certain area or period during one periodic unit (product of (A) and (B)).

voltage on-off is fixed. Therefore, the timing when a particle passes a specific location in the laddertype electrode region is ascertained and can be controlled by changing the phase of the signal. In addition, as the time required for the particles to pass through one periodic unit of the electrodes is equal to the period of the on-off cycle, the particle velocity can be controlled.

In this study, we evaluated the probability density functions of the timing, spacing, and velocity for polystyrene beads and Jurkat cells measured at the inlet and outlet of ladder-type electrodes and achieved accurate timing and alignment control of the particles and cells. We measured and conducted numerical simulation to evaluate the particle motions flowing through the electrode region. Particle velocity, DEP force, flow drag force, and the effects of the periodic cycle of DEP force were analyzed in relation to the particle streamwise position or time to show the contributions of the acceleration and deceleration regions and explain the mechanism of the present technique.

\section{Principle of Timing Control and Alignment by Applying Periodic Forces}

When particles simply flow in the microchannel, the distance between each particle remains constant. If additional force is exerted on the particles in steady state, they are accelerated or decelerated until the particle velocity matches that at which the Stokes force balances the external force and the distance between the particles is similarly maintained. To make the particles align in the streamwise direction 
with even spacing between each particle, additional force needs to be exerted on them; the magnitude of this force depends on the deviation from the aligned position (equilibrium state). We apply two techniques to realize this function: (1) Generate regions of DEP forces periodically in the streamwise direction to accelerate and decelerate particles. (2) Activate the DEP forces in cycles and arrange periods at which no force is exerted on the particles. Combining these two techniques, particles that deviate from the equilibrium state in which the work performed on a particle in one periodic cycle is zero, will experience additional acceleration and deceleration forces.

Figure 2 shows a schematic diagram of how DEP forces are exerted on particles and how particles are controlled to move to the equilibrium state. We assume for simplicity that the force that is exerted on the particles is distributed periodically in the streamwise direction in the sinusoidal wave, and accelerate and decelerate the particles in each region. This structure is similar to a standing wave generated in the channel. The force is activated periodically (in the figure, the duty ratio is 50\%); thus, the force is exerted on the particle at certain periods.

Particles experience acceleration and deceleration forces periodically as they flow through this region. The work applied to particles during one periodic unit for space or time differs depending on the positon (time phase) of the particles relative to the force distribution (force-activated/non-activated cycle). The force distribution of this period can be expressed as the product of the force spatial distribution and force applied period, as shown in the figure, and the work is the integrated value of the force. If the work performed on the particles is positive, they will accelerate during the periodic unit, and vice versa. Particles will thus converge to a specific position (equilibrium state) in which the integrated value of the positive and negative forces are balanced, and the work performed in one periodic unit is zero.

As all particles converge to the equilibrium state, the relative time of the particle to the DEP forceactivated period will be the same. This implies that we can predict the time when the particle passes a certain position by referring to the voltage signal applied to produce the DEP force. Similarly, the relative positon of particles to the spatial distribution of the DEP forces will remain the same. Therefore, the distance between each particle is a multiple of the periodic length of the force distribution, $L_{\text {pitch. }}$. Further, because the particle motion for one periodic unit is synchronized with the force-activated/non-activated cycle, the time required for the particle to move length $L_{\text {pitch }}$ is equal to the period of this cycle $t_{\text {on-off. }}$. Therefore, particle velocity $u_{\mathrm{p}}$ is $u_{\mathrm{p}}=L_{\mathrm{p} i t c h} / t_{\mathrm{on}-\mathrm{off}}=L_{\mathrm{pitch}} f_{\mathrm{on}-\mathrm{off}}$, where $f_{\text {on-off }}$ is the frequency of the force-activated/non-activated cycle.

It should be noted that the rate of the period when the force is activated during one periodic cycle $t_{\mathrm{on}} /\left(t_{\mathrm{on}}+t_{\mathrm{off}}\right)$ can be changed. If the rate is small, the work done on the particle per periodical cycle is small, and the total number of periodic units required for the particles to flow through and make their distances and velocity converge to the specific value increases. On the other hand, if the rate increases and approaches one, the work done on each particle becomes equal and the effect of the force 
correcting the relative position (time phase) of the particles decreases.

\section{Experimental Method}

As shown in Fig. 1, ladder and rail shaped electrodes were patterned on the channel bottom wall, and the ground electrode was attached to the top wall covering the whole surface of the top wall. The distance between parallel electrodes was $20 \mu \mathrm{m}$ and the pitch of the crosstie electrodes was $50 \mu \mathrm{m}$. Three inlets were built in the microchannel to generate sheath flow. Particles were supplied from the center inlet to the channel and sheath flow was controlled to roughly guide particles to the inlet of the rail-type electrodes. The rail-type electrodes controlled the spanwise and height positions of the particles to locate them close to the bottom wall and the centerline of the two parallel electrodes. ${ }^{12,} 16$ Particles were then led to the ladder-type electrode region.

AC voltage was applied to the electrodes at a frequency of $10 \mathrm{MHz}$ with a voltage of $V_{\mathrm{p}-\mathrm{p}}=18 \mathrm{~V}$ to generate the electric field and exert the DEP force on the particles. The voltage was turned on and off with a frequency of $f_{\text {on-off }}$ by using the signal burst mode of the function generator. The sign of the DEP force depends on the frequency of the AC voltage and the ratio between the electric permittivity of the fluid and the particle. In this study, the working fluid was phosphate buffered saline (PBS) and the particles were polystyrene beads and Jurkat cells. In this case, negative DEP force was exerted on the particles, which made them move away from the edges of the electrodes at which the high electric field intensity gradient was generated. ${ }^{12}$

Electrodes were patterned on glass substrates using sputtering and lithographical lift-off processes. The electrodes were made of platinum with titanium bonding layer located between the glass and $\mathrm{Pt}$ electrode. An SU-8 (MicroChem Co.) layer was spin-coated onto the glass substrate and a microchannel was patterned onto the layer using photolithography. The other glass substrate was attached to the other side of the SU-8 layer using thermal bonding. Electrodes were wired with several wiring lines patterned on the bottom wall. $\mathrm{SiO}_{2}$ layer was coated on the wiring areas as electric insulator to avoid the DEP forces of the electric field generated by the wiring lines affecting the particle motion. (See Micro-device fabrication method in Supporting Information (SI) for details.)

Fluid was supplied to the microchannel using a pump that is driven by compressed air. The flow rate and cross-sectional average velocity were varied in the range $Q=5.5-6.8 \mu \mathrm{L} / \mathrm{min}$ and $u_{\mathrm{m}}=9.0-11.0$ $\mathrm{mm} / \mathrm{s}$, respectively.

The motions of the particles were measured based on epi-observation using a microscope (Olympus Co., IX-71) and a high-speed video camera (Vision Research Co., Phantom V1610). Objective lenses with different magnifications were used to measure particle movements for long and short distances. The respective spatial resolution of the images for combination of the camera and these lenses were 
$1.40 \mu \mathrm{m} /$ pixel and $0.68 \mu \mathrm{m} /$ pixel. Bright-field images of particles were recorded and the position and velocities measured using particle motion tracking software Cosmos Move-tr/2D (Library Co.). The maximum time of each sequential measurement and analysis was 1-2s due to the limitation of the camera memory.

Polystyrene micro-particles with size 8, 10 and $12 \mu \mathrm{m}$ (ThermoFisher Scientific Co., 4208A, 4210A, 4212A) and Jurkat cells were used for measurements. Jurkat cells (RBC0806) was provided by the RIKEN BRC through the National Bio-Resource Project of the MEXT/AMED, Japan.

The working fluid was phosphate buffer saline (PBS: Calbiochem, 6500). Sodium lauryl sulfate (Nacalai tesque Co., 31606-62) was mixed with the fluid in the particle case to achieve $0.1 \mathrm{wt} \%$ and thereby preventing the particles adhering to the channel wall. Micro-particles were suspended in the fluid supplied from the center inlet with a volume rate of $0.2 \%$. In the case of Jurkat cells, cells were washed twice with PBS and was supplied from the center inlet with a volume rate of $0.315 \%$.

\section{Numerical Method}

Three-dimensional numerical simulation for the motion of particles of $12 \mu \mathrm{m}$ diameter flowing along the ladder-type electrode was carried out to evaluate the performance of the position control and alignment of particles. An electric field was obtained by solving Poisson equation for the relationship between electric potential and charge using COMSOL Multiphysics (ver. 4.3a: COMSOL Inc.). The electric field distribution obtained by the computation was used to calculate the DEP force $F_{\text {DEP }}$ by applying the multipole method with Clausius-Mossotti (CM) function. ${ }^{53,54}$ The multipole method derives the electric field distribution generated around the particle by applying multiple dipoles. It can express the electric field more accurately than models that use the first order CM function. This is important particularly in cases where the scale of the electric field distribution is equivalent to the particle size. ${ }^{12}$

Particle motion was calculated by applying the $F_{\mathrm{DEP}}$ obtained by the model and the hydrodynamic force to the equation of motion. Equations of motion for translation and rotation were both considered in this study. The hydrodynamic forces acting on the particles were based on Stokes force for spheres, to which the models of Goldman et al. ${ }^{55,56}$, and Cooley and O'neill ${ }^{57}$ considering the effects of the velocity gradient and the wall were applied (see Numerical method in SI for details).

\section{Results and Discussion}

Motion and alignment characteristics of particles. The motion characteristics of particles converging to periodic motions in equilibrium state is evaluated in this section. Figure 3(a) shows the $F_{\text {DEP }}$ distributions when voltage is applied to the ladder-type electrode region obtained by the 


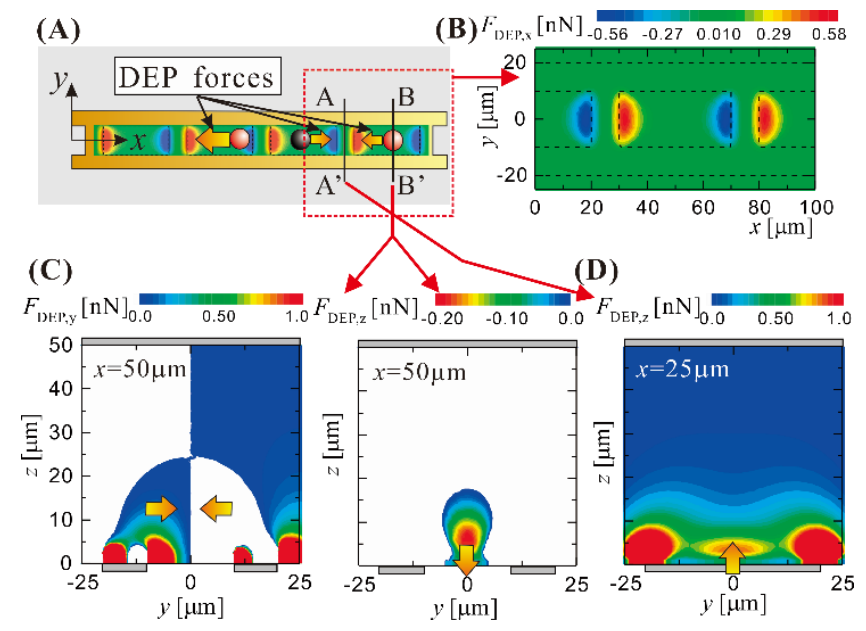

Figure 3. Contour maps of DEP force $F_{\mathrm{DEP}}$ generated by ladder-type electrodes. (A) Schematic of ladder-type electrode and $F_{\mathrm{DEP}, \mathrm{x}}\left(x\right.$ component of $F_{\mathrm{DEP}}$ ) contour distribution. (B) $F_{\mathrm{DEP}, \mathrm{x}}$ in $x-y$ plane at $z=6 \mu \mathrm{m}$. (C) $F_{\mathrm{DEP}, \mathrm{y}}\left(y\right.$ component of $\left.F_{\mathrm{DEP}}\right)$ in $y-z$ plane at location $x=50 \mu \mathrm{m}$. (D) $F_{\mathrm{DEP}, \mathrm{z}}(z$ component of $\left.F_{\mathrm{DEP}}\right)$ in $y-z$ plane at locations $x=25$ and $50 \mu \mathrm{m}$.

computation. The $x, y$, and $z$ components of $F_{\mathrm{DEP}}$ on the $x-z$ plane and the $y-z$ plane are shown in the figure, respectively. $F_{\mathrm{DEP}, \mathrm{x}}$ of the positive and negative values are distributed in the streamwise direction, periodically. The $F_{\mathrm{DEP}, \mathrm{x}}$ will directly accelerate and decelerates the particles in the streamwise direction when the force is activated. $F_{\mathrm{DEP}, \mathrm{y}}$ exhibits a distribution symmetric to the centerline of the ladder-type electrode, and particles are driven to focus on the centerline. $F_{\mathrm{DEP}, \mathrm{z}}$ distributions depicted in (d) exhibit a negative value in the area in the middle of the crosstie electrodes, and a positive value in the area near the crosstie electrodes. Therefore, particles receive forces to approach to and deviate from the channel bottom walls periodically as they flow over the electrodes. The height position of the particles and trajectory is same for each particle but the periodical motion may affect the applicability to 3-D focusing of particles and cells in microfluidic devices.

Figure 4 shows the particle streamwise velocity $u_{\mathrm{p}}$ distributions obtained by the computations of particle motion along the ladder-type electrode and by the measurements. The channel flow rate in the computation and experiment was $Q=6.2 \mu \mathrm{L} / \mathrm{min}$ and cross-sectional average velocity was $u_{\mathrm{m}}=10$ $\mathrm{mm} / \mathrm{s}$. The on-off frequency of the voltage was $125 \mathrm{~Hz}$.

Figure 4(a) depicts the particle velocity $u_{\mathrm{p}}$ distribution along the streamwise position $x_{\mathrm{p}}$ for several periodic units. The dashed line in the figure shows the results of simulation whereas the solid lines represent the results of measurements for four different particle samples. Figure 4(b) shows the results of measurement in the area of one periodic unit using a greater magnification objective lens. The location of the particle center for the images interrupted by the electrodes was estimated by complementing the whole particle shape from the measured one. The fluctuation observed in the measurement values is attributed to the low resolution and performance of the applied image analysis 


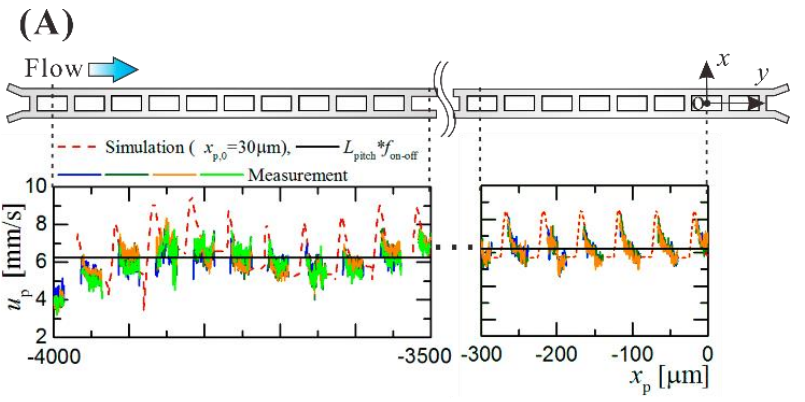

(B)

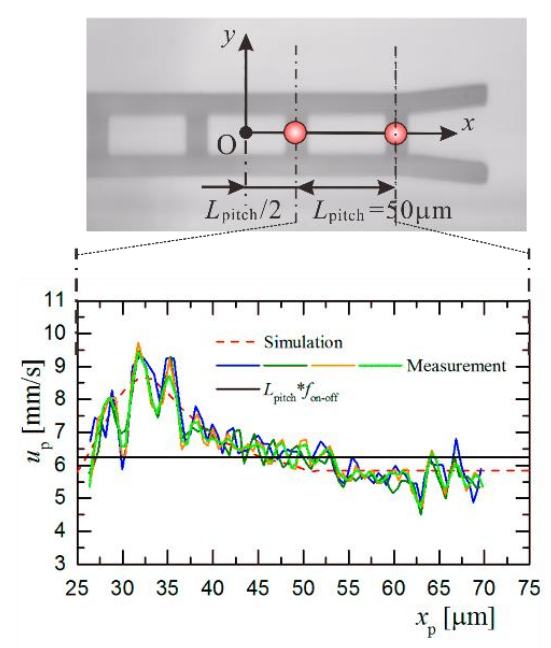

Figure 4. Streamwise velocity $u_{\mathrm{p}}$ distributions of particles at inlet and after converging to a specific velocity in equilibrium state (aligned condition). (A) $u_{\mathrm{p}}$ distributions over several periodic units at inlet and near outlet regions of ladder-type electrode. (B) $u_{\mathrm{p}}$ distribution over one periodic unit in equilibrium state.

for particle velocity and position.

At the inlet of ladder-type electrode region, $u_{\mathrm{p}}$ shows deviation from the equilibrium state $\left(=L_{\mathrm{pitch}} \cdot f_{\mathrm{on}-}\right.$ off) attributed to the randomness of motion characteristics of the particles located upstream. $u_{\mathrm{p}}$ of each particle, however, starts to converge to the equilibrium state as it flows along the ladder-type electrodes and show a periodic pattern in the region near the outlet as shown in Fig. 4(a).

The periodic pattern presented in Fig. 4(b) shows that $u_{\mathrm{p}}$ increase as the particle crosses the crosstie electrode, shows a maximum peak and gradually decrease in the downstream side. $u_{\mathrm{p}}$ then becomes constant in the area of $55<x_{\mathrm{p}}<75 \mu \mathrm{m}$. These areas represent the regions where the acceleration and deceleration forces, and no-force are exerted on the particles.

To discuss these relationships in detail, we will evaluate the particle position, velocity and force exerted on the particles on the basis of the numerical results. Figure 5 shows the distributions of $F_{\mathrm{DEP}, \mathrm{z}}$, $F_{\text {DEP, }}$, particle height position $z_{\mathrm{p}}$, force applied to particle by the flow $F_{\mathrm{Flow}, \mathrm{x}}$, and particle streamwise velocity $u_{\mathrm{p}} . F_{\mathrm{x}}$ is the streamwise component of the total force applied to the particles $\left(F_{\mathrm{DEP}, \mathrm{x}}+F_{\text {Flow, } \mathrm{x}}\right)$. 


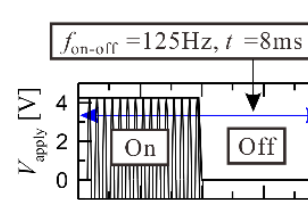

(A)
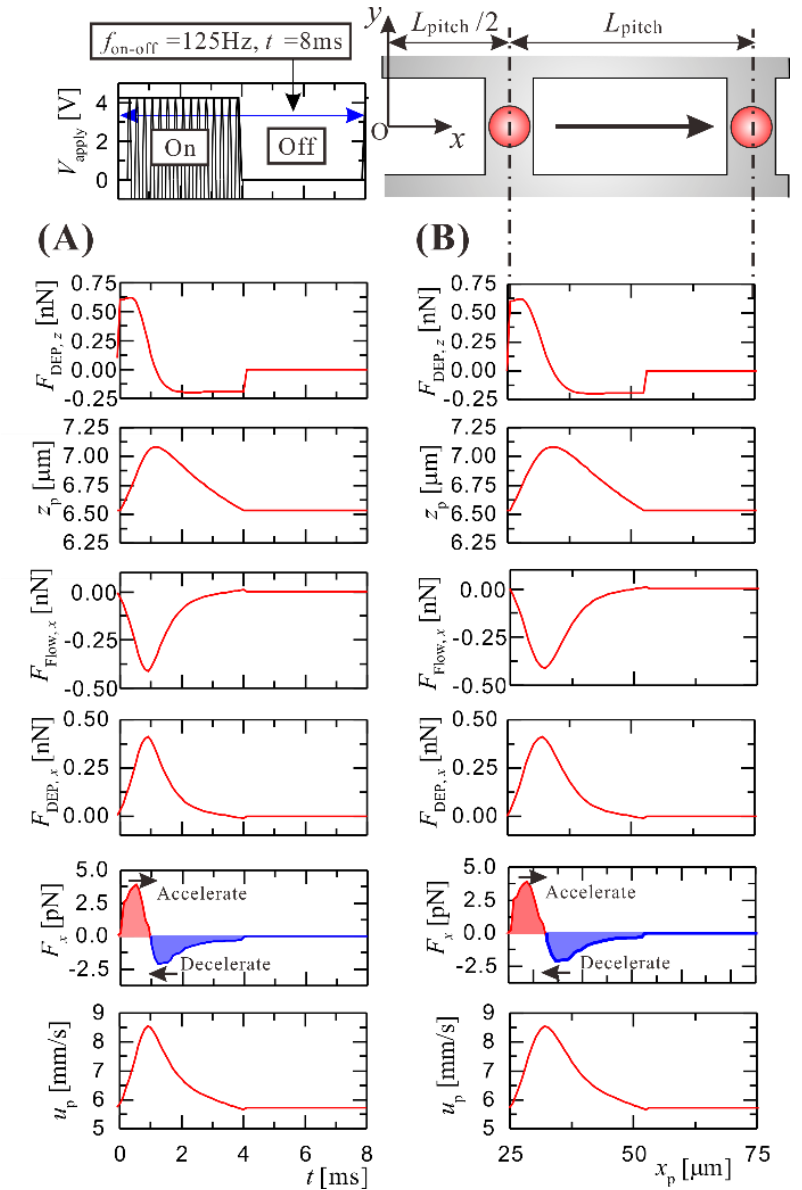

Figure 5. Distributions of DEP forces $F_{\mathrm{DEP}}$, particle height position $z_{\mathrm{p}}$, and particle streamwise velocity $u_{\mathrm{p}}$ of one periodic motion in equilibrium state. $V_{\text {apply }}$ is the AC voltage applied to the electrode with frequency of $f_{\mathrm{V}} . F_{\mathrm{DEP}, \mathrm{x}}$ and $F_{\mathrm{DEP}, \mathrm{z}}$ are the $F_{\mathrm{DEP}}$ components in the $x$ and $z$ directions. $F_{\text {Flow }, x}$ is the component in the $x$ direction of the fluid force exerted on the particle. $F_{x}$ is the total force operating on the particle in the $x$ direction. (A) and (B) are distributions shown in relation to time $t$ and particle streamwise positon $x_{\mathrm{p}}$, respectively.

Figures 5(a) and (b) show the same values of the coordinates in relation to time $t$, and to particle streamwise positon $x_{\mathrm{p}}$. The period when the voltage is applied to the electrodes and the top view of the electrodes are also shown in the figure to associate the timing and location with the aforementioned values.

$F_{\text {DEP,Z }}$ is positive in the area adjacent to the crosstie electrodes and becomes negative in the region located in the middle of the crosstie electrodes. Therefore, particle height position $z_{\mathrm{p}}$ increases near the crosstie electrodes, and with the effect of $F_{\mathrm{DEP}, \mathrm{x}}$ showing a positive value, particles are accelerated in this area. In the downstream region, $z_{\mathrm{p}}$ decreases and the particle moves to the low flow velocity regions which decelerate the particles owing to the fluid drag force as shown by the negative value of $F_{\text {Flow, } x}$. Particles thus accelerate and decelerate during the period when the voltage is supplied 


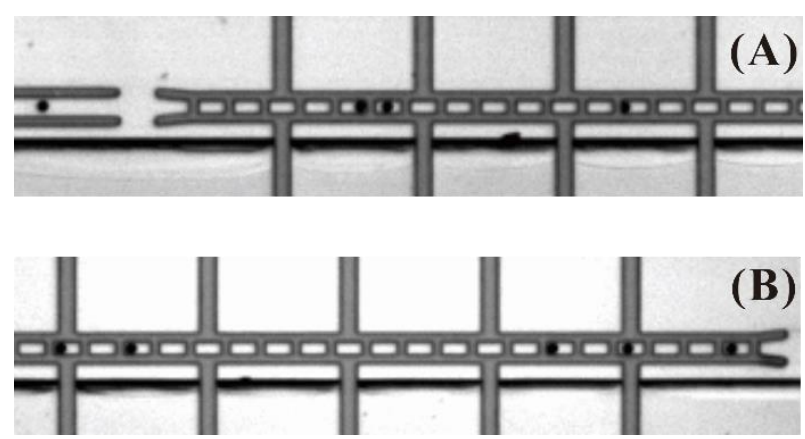

Figure 6. Photographs of particles flowing over ladder-type electrodes. (A) Particles entering ladder-type electrode region. Particles are randomly positioned in the streamwise direction. (B) Particles flowing near the outlet region of the ladder-type electrodes.

exhibiting the $u_{\mathrm{p}}$ distribution shown in Fig. 4(b).

The work done on the particles by the acceleration and deceleration forces balances during the period when force is applied and the total work is zero. If the position of a particle deviates from this equilibrium state, its position relative to the force distribution changes in space and time. If the phase delays or $u_{\mathrm{p}}$ is small, the period at which the positive forces are exerted on particles increases compared with the negative force region during the period when the voltage is applied. If the phase advances or $u_{\mathrm{p}}$ increase, the force activated period will move downstream and negative force due to fluid drag becomes dominant in the period. These additional forces move the particles and its phase to the equilibrium state.

Particle alignment performance-timing, spacing, and velocity. Figure 6 shows photographs of particles with size of $12 \mu \mathrm{m}$ diameter flowing at the inlet and outlet regions of the ladder-type electrode. The distance between the particles entering the region is random. The spanwise and height positions are controlled by rail-type electrodes located upstream of the ladder-type electrodes; however, the relative positions between the particles are random and their streamwise velocities are not controlled. As the particles flow over the ladder-type electrodes, $F_{\mathrm{DEP}}$ is exerted on the particles and causes them to move to the equilibrium state. The distance between particles is thus

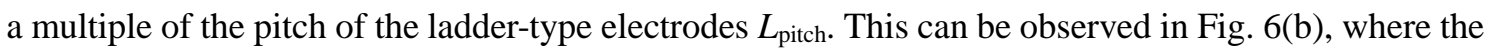
instantaneous positions of the particles are located at the same positon relative to the crosstie electrodes.

Figure 7 shows the probability density distributions of the particle motions measured in the inlet and outlet of the ladder-type electrode region in the microchannel. Figure 7 is the distribution of $\delta t_{\mathrm{s}}$, which is the deviation from the timing for which the particles should exit the ladder-type electrode region. As previously mentioned, the timing when the particle crosses a certain position is synchronized with the phase of the $F_{\mathrm{DEP}}$ on-off cycle. This timing can be controlled by changing the phase of the applied voltage signal as it is linked to the $F_{\mathrm{DEP}}$ cycle (see Reading and controlling the timing of particles in 

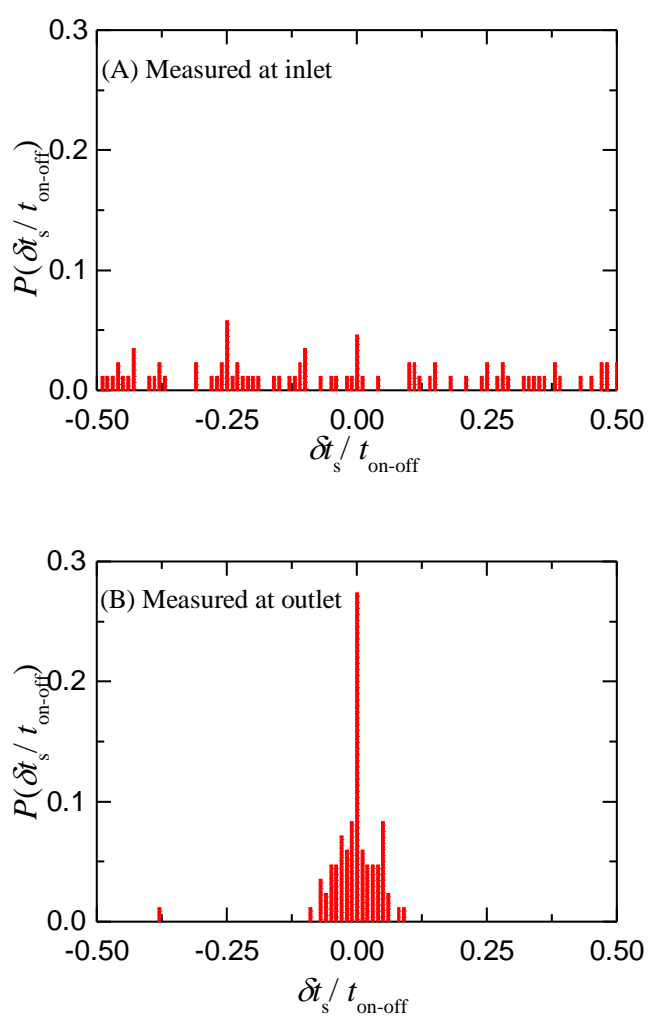

Figure 7. Probability density distributions of particles measured at (A) inlet $(n=84)$ and (B) outlet $(n=86)$ of ladder-type electrode region. PDF is for time deviation from the controlled timing of the particles to flow through the inlet or outlet $\delta t_{\mathrm{s}} . t_{\mathrm{on}-\mathrm{ff}}$ is the period of the particle to flow over one periodic unit of the ladder-type electrode. $t_{\text {on-ff }}$ corresponds to the reciprocal number of voltage onoff frequency, $1 / f_{\text {on-off. }}$

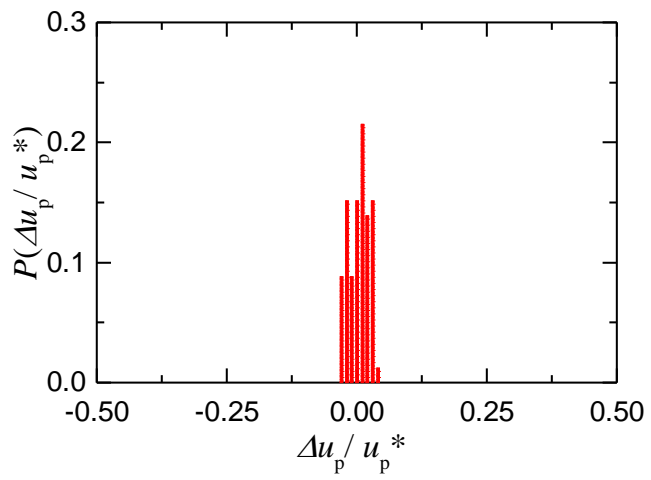

Figure 8: Probability density function of deviation of particle velocities from target value $u_{\mathrm{p}}{ }^{*}=L_{\mathrm{p} i t c h} f_{\mathrm{on}-\mathrm{off}}$ measured at outlet of ladder-type electrode region $(n=86) . \Delta u_{\mathrm{p}}$ is the value of $u_{\mathrm{p}}-u_{\mathrm{p}}{ }^{*}$.

SI for further data). $\delta t_{\mathrm{s}}$ is the deviation from this controlled timing. This value is also equivalent to the deviation of the time difference between particles from the period of the on-off cycle $t_{\text {on-ff. }} n$ shown in the caption represents the number of the measured particles. 

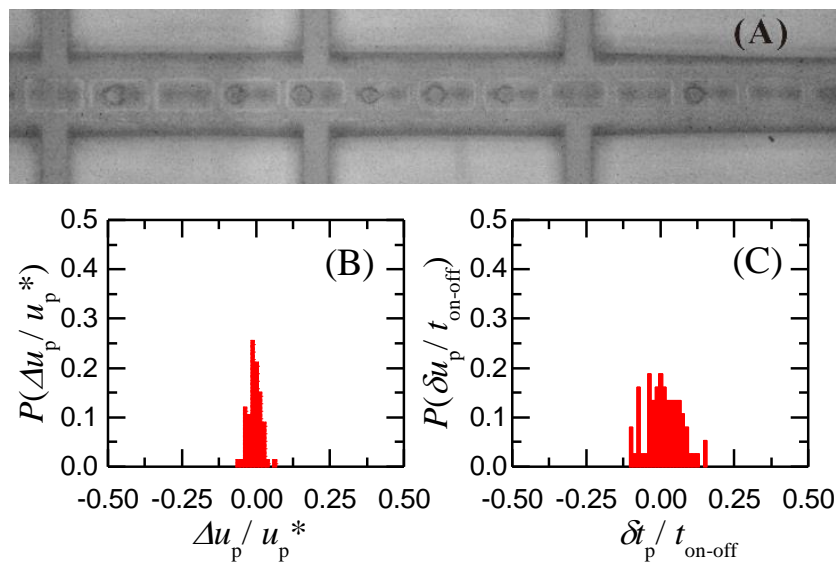

Figure 9: (A) Photograph of Jurkat cells flowing and aligned in the downstream part of the laddertype electrode region, and probability density distribution for variation of the (B) velocity and (C) timing control of the Jurkat cells passing a specific location in the outlet region $(n=63)$.

The graph of the inlet region drawn in Fig. 7(a) shows a random distribution. This indicates that the particles entering the inlet region are located randomly in terms of time and also by relative streamwise positions. However, in the outlet region shown in Fig. 7(b), a peak is observed at $\delta t_{\mathrm{s}} / t_{\mathrm{on}-\mathrm{ff}}=0$. The deviation of the distribution is less than $\pm 5 \%$. This shows that particles flow with equal distance and time, and at timing synchronized with the on-off cycle. In the case of the time interval of the particles $\Delta t_{\mathrm{p}}$, the probability density also shows a distribution for random positioning in the inlet region, while peaks with time interval of $t_{\text {on-off }}=1 / f_{\text {on-off }}$ (or its multiple values) were observed in the outlet region (see Fig. S5 in SI).

Figure 8 shows $\delta u_{\mathrm{p}}$, which is the deviation of the particle velocities measured at the outlet of the ladder-type electrode region from the target value $u_{\mathrm{p}}{ }^{*}=L_{\text {pitch }} f_{\text {on-off. }}$. A peak is apparent at $\delta u_{\mathrm{p}}=0$ and deviation from $\delta u_{\mathrm{p}}=0$ is less than $\pm 3 \%$. Thus, $u_{\mathrm{p}}$ converges to the equilibrium state of $L_{\text {pitch }} f_{\text {on-off. }}$

Measurement using Jurkat cells and particles with a variation in size. Measurement of the timing control and alignment using Jurkat cells was conducted to evaluate the performance of the present method on living cells. Figure 9 shows the snapshot of Jurkat cells aligned in the ladder-type electrode region, and the probability density distribution of the timing and velocity measured at a certain positon in the electrode region with on-off frequency of the applied voltage of $125 \mathrm{~Hz} . n$ is the number of cells measured. The cells are aligned by the DEP force and the distributions of $\Delta u_{\mathrm{p}} / u_{\mathrm{p}}{ }^{*}$ and $\delta t_{\mathrm{s}} / t_{\text {on-off }}$ concentrates at zero showing that the velocity and timing are successfully controlled in this case also. Compared to the particle case shown in Fig. 7, however, the deviation of the distributions increases. This could be attributed to the variation in the size, shape and electric properties of the Jurkat cells.

The size of the particle and cell in the sample fluid may not be the same and may exhibit variations in practical situations. As the DEP force is proportional to the cube of the diameter of the particle, the 

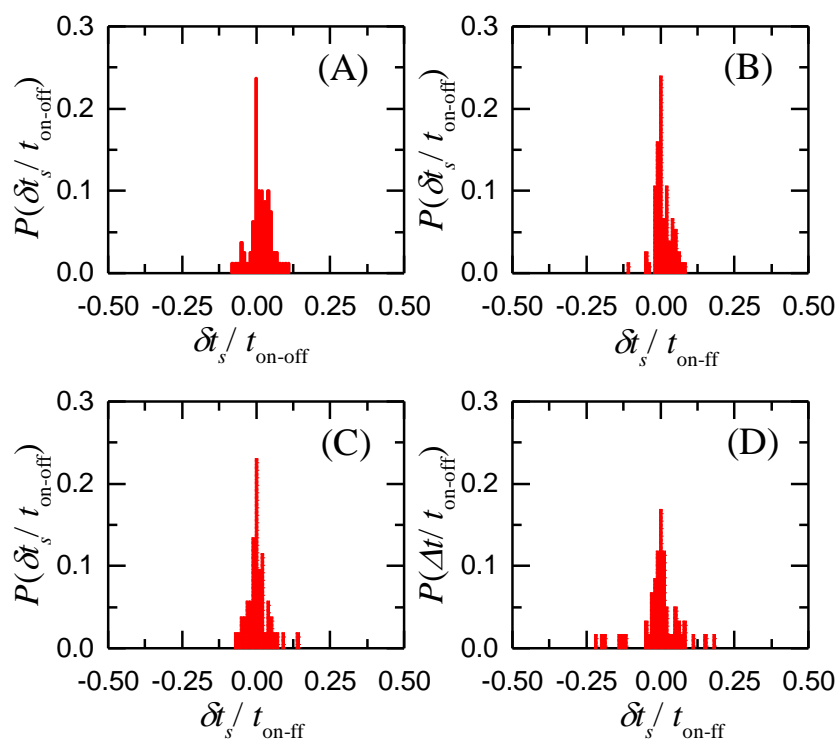

Figure 10: Probability density distribution for variation of the time of the particle passing a specific location in the outlet region of the ladder-type electrodes for particles with variation in size: (A) $d$ $=8 \mu \mathrm{m}(n=80),(\mathrm{B}) d=10 \mu \mathrm{m}(n=57),(\mathrm{C}) d=12 \mu \mathrm{m}(n=58)$, and (D) Mixture $(n=58)$.

magnitude of the DEP force will change in the ladder-type electrode region depending on the particle size, and can affect the performance. In addition to this, the particles flow along the electrode in the wall near region changing its height periodical as shown in Fig. 4. Therefore, the particle motion is affected by the velocity profile. The particle velocity as well as the timing can be controlled for various flow rates; however, an optimum flow velocity exists when consideration is given to the stability and performance of the particle velocity convergence. The optimum flow velocity exhibited a linear relationship with the controlled particle velocity $u_{\mathrm{p}}=L_{\text {pitch }} f_{\text {on-off. }}$. (see Stability of particle motion and flow velocity effects on alignment performance in SI.) Thus, the conditions to control the timing and align the particle using the present method has some limitation. It is, therefore, important to evaluate the accuracy of the present method when some variation in size exists in the particle of interest.

We measured the probability density distribution for particles with diameters 8,10 , and $12 \mu \mathrm{m}$ assuming some deviation in size appears in the particles. Fluids with each particle suspended independently, and for fluids comprising mixtures of these particles were evaluated. The on-off frequency of the voltage was $125 \mathrm{~Hz}$. Figure 10 shows the probability density distribution for the time interval $\delta t_{\mathrm{s}} . n$ shown in each figure are the number of the measured particles. In Figs. 10 (a)-(c), the distributions of $\delta t_{\mathrm{s}} / t_{\mathrm{on}-\mathrm{ff}}$ for the particle diameters 8,10 , and $12 \mu \mathrm{m}$ show a maximum peak at zero and the variation falls in the rage of $\pm 10 \%$. In the same manner, the distributions of $\delta t_{s} / t_{\text {on-ff }}$ concentrate at zero for the mixture of particles, as shown in Fig. 10 (d). This shows that the timing and spacing can be controlled to the same specific value for particles with size variation of at least several tens of percent (see PDF of velocities for particle with different diameters in SI for the velocity data).

The principle of the alignment is based on the balance between the work that accelerates and 
decelerates the particles during the period when the electric field is turned on and the DEP force is exerted on the particles. When the particles converge to the equilibrium state, the total work in one periodic unit becomes zero unless additional force with dissimilarity produced by external factors is exerted on the particle. This principle can be applied to particles of different sizes, and if the force distribution shows similarity among each particle, they will converge to the same position.

As the timing of the particles crossing a certain position in the ladder-type region and time interval of the particles converged to a specific value for the particles of diameter 8,10 , and $12 \mu \mathrm{m}$, the next question is whether we can specify this timing from the voltage signal. The phase difference between the time the particles cross the streamwise center of one periodic unit of the ladder-type electrode and the time for the rising edge of the voltage on-off were examined for these particles. The peaks of the phase difference distributions for particles of diameter 8,10 , and $12 \mu \mathrm{m}$ were located at $0.59 t_{\text {on-off, }}$, $0.53 t_{\mathrm{on}-\mathrm{off}}$, and $0.6 t_{\mathrm{on}-\mathrm{off}}$, respectively (see Reading and controlling the timing of particles in SI for further data). Therefore, the relative time from the voltage signal for a particle at a specific location can be defined within a variation of less than $10 \%$ for particles size varying in this certain range.

This discussion may be extended a little further for particles that have various electrical properties and shapes. If the sign is the same and only the magnitude of the DEP force differs in these cases so that the forces have a similar distribution, particles converge to the same equilibrium state in which the timing, spacing and velocities will match to the specific phase against the voltage on-off, $L_{\text {pitch }}$, and $L_{\text {pitch }} \cdot f_{\text {on-ff }}$, respectively. However, as the particle motion is also affected by the fluid dynamic force, the phase of the timing can change and influence the performance of the timing control if the force distribution shows a significant dissimilarity among the particles.

\section{Conclusions}

This paper proposed a technique in which the timing, spacing, and velocity of particles flowing in a microchannel can be controlled by using the dielectrophoretic (DEP) forces generated by ladder-type electrodes. The particle acceleration and deceleration forces generated by the DEP force and the fluid drag force are distributed in a standing wave form. The force is activated periodically in time and is applied to the particles intermittently. This intermittence is the key factor to allow a phase shift in time and space for the force applied period when the particle deviates from the equilibrium state and make the particle return to the equilibrium state. Particles timing were controlled and were aligned in the measurement with even time interval and distance equal to reciprocal of the frequency of the voltage intermittence $f_{\text {on-off }}$ and the electrode pitch $L_{\text {pitch }}$, respectively. This convergence to equilibrium state was observed in the present measurement and numerical results. The accuracy was demonstrated by the probability density function of the time-deviation from the controlled timing and particle velocity showing a peak distribution with deviation less than $\pm 5 \%$ and $\pm 3 \%$, respectively in $12 \mu \mathrm{m}$ particle case. 
The measurement also showed that the present method can control the timing and velocity of Jurkat cells. The particles of diameter 8,10 , and $12 \mu \mathrm{m}$ converged to the same equilibrium state for timing and spacing, showing that we can align a mixture of particles with a certain degree of variation in sizes if the force distribution shows similarity.

On the other hand, as we use the electrodes to produce the DEP forces, if $L_{\mathrm{p} i t c h}$ increases, more than one particle may converge to the same position. Therefore, evaluation of these effects should be considered as future work to improve the present technique. In contrast, we can apply multiple lines in a microchannel or multiple channels to control different particles in different lines and match or change the timing when they locate at a certain position or merge in the channel. Further, there are other sources than DEP force that can generate periodic force distributions in space and time. The concept of the present technique can employ those alternative forces and be applied not only to microfluidics but also to various fields and applications.

\section{Acknowledgement}

This work was supported by the Japan Society for the Promotion of Science KAKENHI Grant Number 17K18841, and partially by Micro/Nano Fabrication Hub in Kyoto University of "Low-Carbon Research Network" funded by the Ministry of Education, Culture, Sports, Science and Technology (MEXT), Japan.

\section{Associated Content}

\section{Supporting Information}

Additional numerical method details, fabrication method of the micro-device, reading and controlling the timing of particles, probability density functions of time interval of particles, stability of particle motion and flow velocity effects on alignment performance, and performance of velocity control for particles of different diameters.

Video S1: The movie of $12 \mu \mathrm{m}$ particles aligned and flowing in the ladder-type electrode.

\section{References}

1. Baroud, C. N.; Delville, J-P.; Gallaire, F.; Wunenburger, R. Thermocapillary valve for droplet production and sorting, Physical Review E. 2007, 75, 046302.

2. Kulrattanarak, T.; van der Sman, R.G.M.; Schroën, C. G. P. H.; Boom, R. M. Classification and evaluation of microfluidic devices for continuous suspension fractionation. Adv. Colloid Interface Sci. 2008, 142, 53-66.

3. Baret, J-C.; Miller, O. J.; Taly, V.; Ryckelynck, M.; El-Harrak, A.; Frenz, L.; Rick, C.; Samuels, M. L.; Hutchison, B. H.; Agresti, J. J.; Link, D. R.; Weitz, D. A.; Griffiths, A. D. Fluorescence- 
activated droplet sorting (FADS): efficient microfluidic cell sorting based on enzymatic activity, Lab Chip. 2009, 9, 1850-1858.

4. Guo, F.; Ji, X-H.; Liu, K.; He, R-X.; Zhao, L-B.; Guo, Z-X.; Liu, W.; Guo, S-S.; Zhao, X-Z. Droplet electric separator microfluidic device for cell sorting. Appl. Phys. Lett. 2010, 96, 193701.

5. Bhagat, A. A.; Bow, H.; Hou, H. W. Microfluidics for cell separation. Med. Biol. Eng. Comput. 2010, 48, 999-1014.

6. Riordon, J.; Nash, M.; Calderini, M.; Godin, M. Using active microfluidic flow focusing to sort particles and cells based on high-resolution volume measurements. Microelectronic Engineering. 2014, 118, 35-40.

7. Sajeesh, P.; Sen, A. K. Particle separation and sorting in microfluidic devices: a review. Microfluid and Nanofluid. 2014, 17, 1-52.

8. Faigle, C.; Lautenschläger, F; Whyte, G.; Homewood, P.; Martin-Badosa, E.; Guck, J. A monolithic glass chip for active single-cell sorting based on mechanical phenotyping. 2015, 15, 1267-1275.

9. Teste, B.; Jamond, N.; Ferraro, D.; Viovy, J-L.; Malaquin, J. Selective handling of droplets in a microfluidic device using magnetic rails. Microfluid and Nanofluid. 2015, 19, 141-153.

10. Shields IV, C. W.; Reyes, C. D.; López, G. P. Microfluidic cell sorting: a review of the advances in the separation of cells from debulking to rare cell isolation. Lab Chip. 2015, 15, 1230-1240.

11. Tatsumi, K.; Kawano, K.; Okui, H.; Shintani, H.; Nakabe, K. Analysis and measurement of dielectrophoretic manipulation of particles and lymphocytes using rail-type electrodes. Medical Engineering and Physics. 2016, 38, 24-32.

12. Obexer, R.; Pott, M.; Zymer, C.; Griffiths, A. D.; Hilvert, D. Efficient laboratory evolution of computationally designed enzymes with low starting activities using fluorescence-activated droplet sorting, Protein Engineering, Design \& Selection. 2016, 29, 355-366.

13. Yan, S.; Zhang, J.; Yuan, D.; Li, Weihua, L. Hybrid microfluidics combined with active and passive approaches for continuous cell separation. Electrophoresis. 2017, 38, 238-249.

14. Chen, T.; Wu, T.-H.; Kung, Y.-C.; Teitell, M. A.; Chiou, P.-Y. 3D pulsed laser-triggered highspeed microfluidic fluorescence-activated cell sorter. Analyst. 2013, 24, 7308-7315.

15. Gawad, S.; Schildb, L.; Renaud, Ph. Micromachined impedance spectroscopy flow cytometer for cell analysis and particle sizing. Lab Chip. 2001, 1, 76-82.

16. Sun, T.; Green, N.G.; Gawad, S.; Morgan, H. Analytical electric field and sensitivity analysis for two microfluidic impedance cytometer designs. IET Nanobiotechnol. 2007, 1(5), 69-79.

17. Cheung, K. C.; Di Berardino, M.; Schade-Kampmann, G.; Hebeisen, M.; Pierzchalski, A.; Bocsi, J.; Mittag, A.; Ta'rnok, A. Microfluidic impedance-based flow cytometry. Cytometry, Part-A. 2010, 77A, 648-666.

18. Lagus, T. P.; Edd, J. F. High-throughput co-encapsulation of self-ordered cell trains: cell pair 
interactions in microdroplets. RSC Advances. 2013, 3, 20512-20522.

19. Abate, A. R.; Chen, C-H.; Agresti, J. J.; Weitz, D. A.; Beating Poisson encapsulation statistics using close-packed ordering. Lab Chip. 2009, 9, 2628-2631.

20. Seiffert, S.; Thiele, J.; Abate, A. R.; Weitz, D. A.; Smart microgel capsules from macromolecular precursors. J. Am. Chem. Soc. 2010, 132, 6606-6609.

21. Schoeman, R. M.; Kemna, E. W. M.; Wolbers, F.; van den Berg, A.; High-throughput deterministic single-cell encapsulation and droplet pairing, fusion, and shrinkage in a single microfluidic device. Electrophoresis. 2014, 35, 385-392.

22. Vasdekis, A. E.; Stephanopoulos, G.; Review of methods to probe single cell metabolism and bioenergetics. Metabolic Engineering. 2015, 27, 115-135.

23. Kemna, E. W. M.; Schoeman, R. M.; Wolbers, F.; Vermes, I.; Weitz, D. A.; van Berg, A.; Highyield cell ordering and deterministic cell-in-droplet encapsulation using Dean flow in a curved microchannel. Lab Chip. 2012, 12, 2881-2887.

24. Velasco, D.; Tumarkin, E.; Kumacheva E.; Microfluidic encapsulation of cells in polymer microgels. Small. 2012, 8, 1633-1642.

25. Tumarkin, E.; Tzadu L.; Csaszar, E.; Seo, M.; Zhang, H.; Lee, A.; Peerani, R.; Purpura, K.; Zandstra, P. W.; Kumacheva, E.; High-throughput combinatorial cell co-culture using microfluidics, Inegr. Biol. 2011, 3, 653-662.

26. Edd, J. F.; Di Carlo, D.; Humphry, K. J.; K"oster, S.; Irimia, D.; Weitzb, D. A.; Toner, M. Controlled encapsulation of single-cells into monodisperse picolitre drops. Lab Chip. 2008, 8, 1262-1264.

27. Mazutis, L.; Gilbert, J.; Lloyd, U.; Weitz, D. A.; Griffiths, A. D.; Heyman, J. A.; Single-cell analysis and sorting using droplet-based microfluidics. Nature Protocols. 2013, 8, 870-891.

28. Brouzesa, E.; Medkovaa, M.; Savenellia, N.; Marrana, D.; Twardowskia, M.; Hutchisona, J. B.; Rothberga, J. M.; Linka, D. R.; Perrimonb, N.; Samuelsa, M. L. Droplet microfluidic technology for single-cell high-throughput screening. PNAS. 2009, 106, 14195-14200.

29. Russom, A.; Gupta, A. K.; Nagrath, S.; Di Carlo, D.; Edd, J. F.; Toner, M. Differential inertial focusing of particles in curved low-aspect-ratio microchannels. New J. Phys. 2009, 11, 075025.

30. Oakey, J.; Applegate, R. W.; Arellano, E.; Di Carlo, D.; Graves, S. W.; Toner, M. Particle focusing in staged inertial microfluidic devices for flow cytometry. Anal. Chem. 2010, 82, 3862-3867.

31. Xuan, X.; Zhu, J.; Church, C. Particle focusing in microfluidic devices. Microfluid Nanofluid. 2010, 9, 1-16.

32. Di Carlo, D.; Irimia, D.; Tompkins, R. G.; Toner, M.; Continuous inertial focusing, ordering, and separation of particles in microchannels. PNAS. 2007, 104(48), 18892-18897.

33. Kahkeshani, S.; Haddadi, H.; Di Carlo, D. Preferred interparticle spacings in trains of particles in inertial microchannel flows. J. Fluid Mech Rapids. 2016, 786, R3, doi:10.1017/jfm.2015.678. 
34. Chung, A. J.; Gossett, D. R.; Di Carlo, D. Three dimensional, sheathless, and high-throughput microparticle inertial focusing through geometry-induced secondary flows. Small. 2013, 9, 685690.

35. Ahn, B.; Lee, K.; Lee, H.; Panchapakesan, R.; Xu, L.; Xu, J.; Oh, K. W. Guiding, distribution, and storage of trains of shape-dependent droplets. Lab Chip. 2011, 11, 3915-3918.

36. Gao, Y.; Magaud, P.; Baldas, L.; Lafforgue, C.; Abbas, M.; Colin, S. Self-ordered particle trains in inertial microchannel flows, Microfluid Nanofluid. 2017, 21, 154-164.

37. Zhang, J.; Yan, S.; Alici, G.; Nguyen, N-T.; Di Carlo, D.; Li, W. Real-time control of inertial focusing in microfluidics using dielectrophoresis (DEP). RSC Adv. 2014, 4, 62076-62085.

38. Gascoyne, P. R. C.; Vykoukal, J. Particle separation by dielectrophoresis, Electrophoresis. 2002, 23, 1973-1983.

39. Hughes, M. P.; Strategies for Dielectrophoretic Separation in laboratory-on-a-chip systems. Electrophoresis. 2002, 23, 2569-2582.

40. Ramos, A.; Morgan, H.; Green, N. G.; Castellanos, A. Ac Electrokinetics: A review of forces in microelectrode structures. J. Physics D - Applied Physics. 1998, 31, 2338-2353.

41. Fiedler, S.; Shirley, S. G.; Schnelle, T.; Fuhr, G. Dielectrophoretic sorting of particles and cells in a microsystem. Anal. Chem. 1998, 70, 1909-1915.

42. Morgan, H.; Green, N. G.; Hughes, M. P.; Monaghan, W.; Tan, T. C. Large-area travelling-wave dielectrophoresis particle separator. J. Micromech. Microeng. 1997, 7, 65-70.

43. Cui, L.; Holmes, D.; Morgan, H. The dielectrophoretic levitation and separation of latex beads in microchips. Electrophoresis. 2001, 22, 3893-3901.

44. Kim, Y. W.; Yoo, J. Y. Three-dimensional focusing of red blood cells in microchannel flows for bio-sensing applications. Biosens. Bioelectron. 2009, 24, 3677-3682.

45. Lin, J. T. Y.; Yeow, J. T. W.; Wan, W. Controlling two-dimensional movement of microparticles over an electrode array surface. Biomed Microdevices. 2009, 11, 193-200.

46. Zhu, J.; Tzeng, T-R. J.; Hu, G.; Xuan, X. DC dielectrophoretic focusing of particles in a serpentine microchannel. Microfluid Nanofluid. 2009, 7, 751-756.

47. Laurell, T.; Petersson, F.; Nilsson, A. Chip integrated strategies for acoustic separation and manipulation of cells and particles. Chem. Soc. Rev. 2007, 36, 492-506.

48. Wood, C. D.; Evans, S. D.; Cunningham, J. E.; O’Rorke, R.; Wälti, C.; Davies, A. G. Alignment of particles in microfluidic systems using standing surface acoustic waves. Appl. Phys. Lett. 2008, 92, 044104 .

49. Shi, J.; Ahmed, D.; Mao, X.; Lin, S.-C. S.; Lawit, A.; Huang, T. J. Acoustic tweezers: Patterning cells and microparticles using standing surface acoustic waves (SSAW). Lab Chip. 2009, 9, 28902895.

50. Afshar, R.; Moser, Y.; Lehnert, T.; Gijs, M. A. M. Three-dimensional magnetic focusing of 
superparamagnetic beads for on-chip agglutination assays. Anal. Chem. 2011, 83, 1022-1029.

51. Lu, X.; Liu, C.; Hu, G.; Xuan, X. Particle manipulations in non-Newtonian microfluidics: A review. J. Colloid Interface Sci. 2017, 500, 182-201.

52. Jones, T. B.; Washizu, M. Multipolar dielectrophoretic and electrorotation theory. J. Electrost. 1996, 7, 121-134.

53. Washizu, M.; Jones, T. B. Multipolar dielectrophoretic force calculation. J. Electrost. 1994, 33, 187-198.

54. Goldman, A. J.; Cox, R. G.; Brenner, H. Slow Viscous motion of a sphere parallel to a plane wall I: Motion through a quiescent fluid. Chem Eng. Sci. 1967, 22, 637-651.

55. Goldman, A. J.; Cox, R. G.; Brenner, H. Slow viscous motion of a sphere parallel to a plane wall II: Couette flow. Chem Eng. Sci. 1967, 22, 653-660.

56. Cooley, M. D. A.; O'neill, M. E. On the slow motion generated in a viscous fluid by the approach of a sphere to a plane wall or stationary sphere. Mathematica. 1969, 16, 37-49. 